
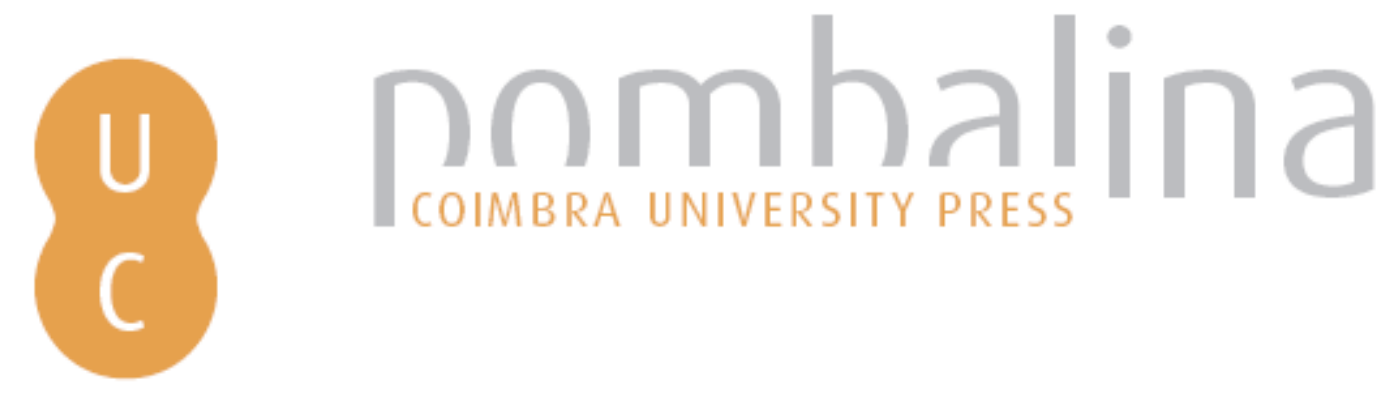

\title{
La giustizia nel Grillo e la conclusione del dialogo
}

Autor(es): Casanova, Angelo

Publicado por: Imprensa da Universidade de Coimbra

URL

persistente:

URI:http://hdl.handle.net/10316.2/32882

DOI:

DOI:http://dx.doi.org/10.14195/978-989-721-012-9_13

Accessed : $\quad$ 26-Apr-2023 02:32:07

A navegação consulta e descarregamento dos títulos inseridos nas Bibliotecas Digitais UC Digitalis, UC Pombalina e UC Impactum, pressupõem a aceitação plena e sem reservas dos Termos e Condições de Uso destas Bibliotecas Digitais, disponíveis em https://digitalis.uc.pt/pt-pt/termos.

Conforme exposto nos referidos Termos e Condições de Uso, o descarregamento de títulos de acesso restrito requer uma licença válida de autorização devendo o utilizador aceder ao(s) documento(s) a partir de um endereço de IP da instituição detentora da supramencionada licença.

Ao utilizador é apenas permitido o descarregamento para uso pessoal, pelo que o emprego do(s) título(s) descarregado(s) para outro fim, designadamente comercial, carece de autorização do respetivo autor ou editor da obra.

Na medida em que todas as obras da UC Digitalis se encontram protegidas pelo Código do Direito de Autor e Direitos Conexos e demais legislação aplicável, toda a cópia, parcial ou total, deste documento, nos casos em que é legalmente admitida, deverá conter ou fazer-se acompanhar por este aviso.

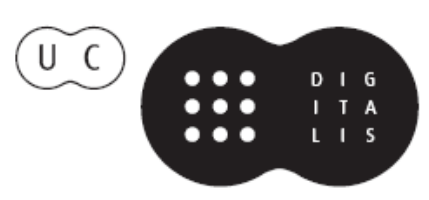




\section{Nomos, Kosmos \& Dike in Plutarch}

José Ribeiro Ferreira, Delfim F. Leão \& Carlos A. Martins de Jesus (eds.) 


\title{
LA GIUSTIZIA NEL GRILLO E LA CONCLUSIONE DEL DIALOGO
}

Angelo Casanova

Università di Firenze

\begin{abstract}
.
The dialogue lacks a section on justice - one of the four virtues according to Plato and Aristotle (justice, fortitude, prudence and temperance). Several scholars believe the work to be incomplete, with the final part being lost; possibly, though, the author may have intended the work to end as we have it, and given up following through with his paradoxical argumentation.
\end{abstract}

Mi sono occupato altre volte del dialogo Bruta animalia ratione uti, detto anche Gryllus, prima ${ }^{1}$ per precisare l'ambientazione per così dire mitica, cioè il suo riferimento ad Omero (la situazione è quella di $O d$. 12.142, non quella di $O d .10 .135-399$, come si dice di solito), poi $^{2}$ per chiarire che l'Ulisse del dialogo presenta caratteri e motivi valorizzati nella tragedia greca del $\mathrm{V}$ secolo. Oggi vorrei richiamare la vostra attenzione sull'ultima pagina del dialogo e sul vecchio, vecchissimo problema se il dialogo sia concluso e perfetto così comè, oppure se il finale sia andato perduto nel corso della tradizione.

Entrambe le opinioni sono già state autorevolmente sostenute da vari studiosi: ma questo incontro, in cui si parla specificamente dell'importanza della giustizia nelle opere di Plutarco, può essere a mio avviso la sede più adatta per una nuova riflessione in proposito.

Come è noto, si tratta di un dialogo mitico - un caso unico nel corpus plutarcheo - in cui Ulisse prega Circe di concedere che i Greci che si trovano presso di lei, trasformati in animali, possano riprendere le sembianze umane e ritornare con lui in Grecia. Circe acconsente a condizione che accettino gli interessati: a tal fine, ella ridà la parola ad uno di essi, un certo Grillo, ora trasformato in maiale, e si allontana (cap. 1). Subito Grillo dichiara che la vita degli animali è migliore di quella degli uomini e, di fronte alle perplessità di Ulisse, si dichiara pronto a dimostrarlo. Nel cap. 3 (986F) egli dichiara di voler cominciare proprio dalle virtù per cui gli uomini ritengono di essere superiori agli animali, e cioè giustizia, assennatezza, coraggio e tutte le altre (); e passa quindi ad argomentare che l'anima degli animali è più adatta a produrre tali virtù, poiché le produce senza bisogno di ordini e insegnamenti (cap. 3). Per la

${ }^{1}$ Nella relazione fatta all'incontro di Lille, edita come A. Casanova 2005: 97-109; cf. A. Casanova 2005b: 121-131.

${ }^{2}$ Nella relazione tenuta al Convegno di Madrid, 21-24 sett. 2005, edita in Ploutarchos n.s. 4, 2006/2007: 19-28. 
precisione, egli lo dimostra anzitutto (cap.4) per il coraggio, poi per i due campi in cui si manifesta il, e cioè prima (A) per la (cap. 5), cioè per la temperanza, o autocontrollo, la capacità di controllare con la mente gli istinti e le voglie, in particolare la cupidigia, la smania di lusso e di profumi (cap. 6), la bramosia e le perversioni in campo sessuale (cap. 7), e infine la smodatezza nel mangiare e nel bere (cap. 8). Segue (B) la trattazione della , ossia dell'intelligenza, intesa anche come capacità di apprendere le arti e le diverse abilità, come ad esempio l'arte medica (cap. 9) e le altre abilità che dimostrano capacità intellettuali e mnemoniche (cap. 10): e anche in queste varie forme di sapienza - egli argomenta - gli animali risultano superiori agli uomini. A questo punto Ulisse obietta che però agli animali manca la , la percezione che esiste la divinità (meglio che la 'conoscenza di Dio'), e Grillo gli dà una risposta bruciante: ; "Allora non dovremmo neppure dire che tu, Ulisse, così saggio e straordinario come sei, sei nato da Sisifo?”. E così il dialogo si chiude bruscamente (o resta interrotto).

La spiegazione più facile è quella data già da K. ZIEGLer 1965: 131: "Grillo ha appena cominciato a rispondere, ma qui si interrompe il testo... certo per una perdita secondaria. Seguivano indubbiamente altre osservazioni come in De soll. anim. 22 e 35-36, e verosimilmente la dimostrazione della degli animali concludeva il dialogo, e Ulisse si dichiarava persuaso". Questa osservazione è stata variamente ripetuta, variata o ridotta, con spiccata differenziazione tra filosofi e letterati: anzi, si può dire che non c'è un altro punto dell'opuscolo in cui la differenza di lettura dei 'filosofi' e dei 'letterati' venga fuori con altrettanta chiarezza. I primi hanno osservato che il dialogo non può finire senza una trattazione della : particolarmente categorici in proposito erano già H. Usener 1887: LXX ("libello nunc mutilato"), U. Dierauer 1977: 191, D. Russell 1993: 348, ma anche F. Becchi nel suo articolo del 1993: 63³. Incerti (o per lo meno problematici) sono, fra gli editori, sia W. C. НеLmbold 1957 che G. Indelli 1995, mentre C. Hubert 1954 mette indicazione di lacuna con tre asterischi. Oltre tutto - si osserva in genere - la trattazione è stata annunciata nel cap. 3 (come s'è visto all'inizio): cf. in particolare J. A. Fernández Delgado 2000: 175. I 'letterati' invece obiettano che con il duro scambio di battute sulla (percezione o nozione degli dei) il dialogo tocca l'apice della tensione ideologica e dialettica, e che ogni altro finale diverso da questo riuscirebbe fatalmente fiacco e opaco. Ad esempio, Helmbold non segna lacuna finale e annota che è ingegnosa l'interpretazione di Reiske, che si può riassumere così: se chi non conosce dio non possiede la ragione, allora tu difficilmente puoi essere detto figlio di Sisifo! ${ }^{1}$ Il sottinteso, naturalmente, è

\footnotetext{
${ }^{3}$ Vd. anche F. Becchi 2000 e F. Becchi 2001; e cf. A. Barigazzi 1992.

${ }^{4}$ Vd. W. C. Helmbold 1957: 532-533, nota $a$ in calce al testo.
} 
che Sisifo era notoriamente ateo, come si argomentava nella tragedia di Crizia intitolato appunto Sisifo, di cui abbiamo un lungo frammento proprio sulla tematica cara a Sisifo che "gli dei sono soltanto un'utile invenzione" $\operatorname{Tr} G F 1, \mathrm{~F}$ 19 Snell). Anche J. A. Fernández Delgado 2000: 171 osserva che il brusco e inatteso finale fa da pendant con l'inizio ex abrupto.

Ora, non c'è dubbio che in un trattato serio sulle virtù dell'uomo è addirittura ovvio che non può mancare la giustizia. Ho già illustrato nell'incontro di Málaga (A. CAsanova 2010: 65-76) che la quadruplice divisione delle virtù proposta da Platone nel IV libro della Repubblica (sapienza, coraggio, temperanza, giustizia), ripresa e definita da Aristotele nell'Etica Nicomachea (nella Metafisica e in altre opere), è di fondamentale importanza in tutta la tradizione successiva. Le quattro virtù platoniche furono riprese anche nel pensiero cristiano e denominate "cardinali" da Sant'Ambrogio e poi ridefinite con maggiore spessore teorico da San Tomaso d'Aquino, che, riprendendo proprio la tradizione aristotelica, distingue nell'ordine le quattro virtù cardinali, cioè 'cardini' dell'etica umana (giustizia, fortezza, prudenza, temperanza), inferiori solo alle virtù teologali, che sono tre (fede, speranza, carità). Ebbene - tornando al nostro opuscolo - il maiale Grillo sa benissimo che, se vuol dimostrare la superiorità degli animali sugli uomini, deve parlare anche di giustizia: anzi, soprattutto di giustizia. Non per caso, nel cap. 3 la pone in prima sede - nell'ordine: giustizia, assennatezza, coraggio e poi tutte le altre (cf. p. 181). Come virtù "cardinale", cioè cardine dell'etica, la giustizia non può mancare.

Ancor di più: in un serrato confronto tra animale e uomo, non può mancare una trattazione di quel delicato problema che è la giustizia relativa tra uomo e animale. Non per caso, ma per ovvia necessità, nell'altro dialogo plutarcheo sugli animali, il cosiddetto De sollertia animalium, la discussione tra due persone vere e reali (Autobulo e Soclaro, rispettivamente padre e amico di Plutarco) sul tema importantissimo "se e quanto gli animali siano forniti di intelligenza e di raziocinio" (per usare ancora le parole di K. Ziegler 1965: 125) porta ben presto (nei primi capitoli) a prendere posizione sul fatto che gli animali hanno intelligenza e ragione, ma non l'aspirazione alla perfezione, cioè la virtù; e si afferma che gli uomini superano gli animali nella facilità dell'apprendere, nell'acume, nel senso di giustizia e nel buon senso comune, mentre gli animali possono essere superiori in grandezza, velocità, acutezza della vista e dell'udito; inoltre fra gli animali è importante l'amore per i figli, ma senza che sia dato loro il vero scopo di questo amore, che è la giustizia (cap. 4). Ma non per questo, si dice al cap. 7, si può - come fanno Stoici e Peripatetici - negare la ragione agli animali per riservare il concetto della giustizia e del diritto sociale al mondo umano, né si deve escludere un rapporto di giustizia e diritto verso gli animali. E questo, naturalmente, è un tema importante, non solo del De sollertia 
animalium, dove si osserva che servirsi degli animali non è ingiustizia di per sé, ma lo diviene se ce ne serviamo in modo cattivo, incurante o crudele (cap. $7)^{5}$, ma anche in un apposito trattato, egualmente plutarcheo, che va sotto il titolo di De esu carnium, purtroppo conservato in modo incompleto e lacunoso, diviso in due parti ${ }^{6}$, cui sono comunque da riferire anche in alcuni frammenti plutarchei attestati da Porfirio ${ }^{7}$. Il tema è in parte ripreso da Plutarco anche in un passo delle Quaestiones Convivales (2.3, 635E-638A)8.

Ebbene, in base a queste considerazioni sembrerebbe chiaro, palese, quasi ovvio che l'opera è incompleta, anzi largamente incompleta, come pensava Ziegler. In proposito però io userei una maggiore prudenza e farei piuttosto un altro tipo di riflessioni.

Il dialogo Gryllus non è un trattato serio, espositivo, dimostrativo; è una esercitazione retorica, divertente e divertita. Non dobbiamo trasformarlo in un trattato a tesi filosofica. $\grave{E}$ un dialogo mitico, del tutto fittizio, e il protagonista è un personaggio a sorpresa, assolutamente inverosimile, e sostiene una tesi retorica, paradossale: chi parla è Grillo, un maiale, e non Plutarco. È una tesi provocatoria.

Io osserverei nel testo due particolari importanti, finora - a mio avviso - non valorizzati abbastanza. Anzitutto il maiale Grillo ha annunciato fin dall'inizio che è pronto a parlare di tutte le virtù, a partire proprio dalla giustizia e a dimostrare la superiorità degli animali in tutte quante le virtù. Poi, per abilità dialettica (o, se vogliamo, sofistica), è partito dalla per sottolineare addirittura che gli animali sono i veri, non gli uomini, che non sanno nemmeno più che cosa sia il coraggio. A mio avviso, è chiaro e scontato che il maiale ha intenzione di parlare di tutte le virtù, ed ha lasciato alla fine la giustizia, perché è l'argomento fondamentale nella contrapposizione fra animali e uomini. $\mathrm{Ma}$ - e qui interviene la seconda osservazione, che non deve sfuggire nella lettura del testo - è Ulisse che nella discussione, impaziente di sottrarsi alla fiumana argomentiva di Grillo, nel tentativo di fermarla e batterla in maniera decisa, interrompe l'esposizione del maiale e getta in campo, all'improvviso, un argomento che dovrebbe a suo avviso troncare la discussione. È l'obiezione che, al di là delle virtù umane caratterizzanti, che noi chiamiamo "cardinali", ci sono

${ }^{5}$ K. Ziegler 1965: 128. Vd. in proposito la Introduzione di Giuseppina Santese, in L. Inglese; G. SAntese i 999: 15 ss. e 23 ss.

${ }^{6}$ Il testo è edito da Helmbold nel vol. XII della Loeb (W. C. Helmbold i 957). Ma vd., più di recente, L. Inglese; G. SAntese i999; e cf. D. Magini 2001.

${ }^{7}$ Fr. 193 SAndBach (vol. VII, pp. 119-122 nell'edizione Teubner; vol. XV, pp. 352-261 nella Loeb). Si veda quanto scritto in proposito da D. Del Corno, nella Introduzione a D. Magini 2001: 37 ss.

${ }^{8}$ Vd. A. Cainzza 2001; S.-T. Teodorsson 1989: 210-232. 
altre virtù, che riguardano la percezione del divino: in termini platonici e plutarchei, c'è la ; in termini aristotelico-tomistici potremmo dire che si accenna alla prima delle virtù teologali (fede, speranza, carità). Ovvero: l'uomo è superiore agli animali perché ha nozione che esistono gli dei. Su questo tutti sembrerebbero d'accordo: anche Epicuro diceva che tutti hanno una (Epic., Ep. ad Men. 123 ss.). Ma non è su questo che vorrei soffermarmi: vorrei invece ripetere che è Ulisse a deviare e fermare il discorso, non Grillo. Dunque è Ulisse che taglia corto e interrompe la dimostrazione prevista o prevedibile, e già programmata. Naturalmente bisogna chiedersi perché. Ovviamente Ulisse è un personaggio, non una persona reale: dunque bisogna chiedersi perché l'autore gli fa dire questo, cioè perché gli fa interrompere la fiumana della dimostrazione. E questa per me - è la base della soluzione dei nostri problemi nell'interpretazione del dialogo plutarcheo. Evidentemente Plutarco vuole che il suo dialogo rimanga un 'divertissement' limitato, e non diventi un lungo trattato dimostrativo, completo ed esaustivo. A mio avviso, qui si tocca il punto qualificante dell'opera: Plutarco non vuole che si arrivi alla questione centrale, nevralgica, importantissima, se c'è la giustizia fra gli animali, e nemmeno all'altra, egualmente campale per lui, quale sia la giustizia verso gli animali (di cui si parla nel De esu carnium). E quindi fa intervenire proprio il protagonista del dialogo, Ulisse, a dare una svolta decisiva alla dimostrazione, imponendo un drastico taglio: al di là delle virtù classiche, c'è l'intuizione teologica! Ciò che manca nel dialogo, cioè il duplice ampio discorso sulla giustizia tra gli animali e verso gli animali, è dunque omesso volontariamente dall'autore, non caduto "per una perdita secondaria" nella tradizione del testo. Questa a me sembrerebbe una conclusione di cui possiamo sentirci abbastanza sicuri.

C'è però da fare una postilla. L'obiezione di Ulisse, indubbiamente brusca e maligna, è respinta con una battuta feroce, non con un argomentazione sofisticamente elaborata e rifinita. La battuta è così tagliente che non è immediatamente chiara nella sua cattiveria, ma è stata ormai adeguatamente spiegata (da Reiske e gli altri) ${ }^{9}$. Atei sono piuttosto certi uomini, non gli animali: ad esempio proprio quel Sisifo che una certa tradizione, maligna, presenta come padre reale di Ulisse per affermare e spiegare la grande intelligenza ed astuzia di lui. La battuta si spiega con riferimento ad una versione del mito sicuramente post-omerica, forse risalente ai Cypria (secondo F. Jounn 1966:279, n. 5), ben attestata nella tragedia, sia in Eschilo (fr. 175 Radt: Armorum iudicium) che in Sofocle (Ai. 190, Phil. 417, 625, 1311; fr. 567 Radt: Syndeipnoi) e in Euripide (IA 524 e 1362; Cycl. 104). Ed

\footnotetext{
${ }^{9}$ Cf. p. 183 e n. 4.
} 
è una versione del mito ripresa e annotata come curiosità antiquaria dallo stesso Plutarco in Aet. Rom. et Gr. 43, 301D ${ }^{10}$, dove si spiega che secondo alcuni Anticlea concepì Ulisse perché violentata da Sisifo appena prima del suo matrimonio con Laerte: Ulisse era dunque intelligente e astutissimo per motivi genetici. A questo allude pesantemente Grillo: o non è vero che è "seme" di Sisifo (cioè non è intelligente), oppure dimentica che proprio il padre naturale a lui attribuito da certuni era notoriamente ateo. La battuta è tagliente, fortemente offensiva, e tronca quindi il discorso. Dopo, non si può immaginare che Ulisse si dichiari convinto, come pensava Ziegler: piuttosto sarà offeso e arrabbiato. Fosse irascibile come Achille, lo potrebbe uccidere all'istante; o forse, più probabilmente, si può immaginare che interrompa qui il suo tentativo di convincerlo e parta senza di lui.

Pertanto, alla luce di queste considerazioni, io non parlerei né di parte mancante perché perduta, né di argomentazione compiutamente e adeguatamente terminata. Di certo l'autore ha concluso bruscamente la sua opera volontariamente, per non portare fino all'assurdo (e alla noia) il suo gioco paradossale. Egli ha esposto a lungo il pensiero di Grillo, ispirato alla tradizione del cinismo ${ }^{11}$, sfruttando anche elementi epicurei ${ }^{12}$, e persino stoici ${ }^{13}$, anche se vari elementi rivelano la preparazione platonica dell'autore ${ }^{14}$, creando un 'pastiche'

${ }^{10}$ Edito nel vol. IV della Loeb, da F. C. BАввітт 1972.

${ }^{11}$ Che la fonte principale sia cinica è ammesso concordemente dalla critica: vd. e.g. R. Hirzel 1895 II: 128 ss.; K. Ziegler 1965: 132; U. Dierauer 1977: 187 ss.; G. Indelli 1995: 25; G. Santese 1999: 44, nn. 115-116, 49; J. A. Fernández Delgado 2000: 172, 176, 180; per un indice dei motivi cinici vd. J. Bergua Cavero 1991: 13-15.

${ }^{12}$ La componente epicurea è stata variamente valutata. Secondo H. Usener 1887: LXX ss. essa percorrebbe il dialogo da un capo all'altro ("argumenta omnia, et saepe quidem verbo tenus, ex Epicuro philosophia petita sunt”). La critica recente ha molto attenuato tale giudizio: G. InDELLi 1995: 22, si limita a rilevare nel dialogo una "presenza di materiale epicureo e venature antiepicuree"; F. ВесCH 2000: 218, n. 103, afferma che "nel Bruta animalia vi è ben poco di epicureo - al di là di espressioni abbastanza generiche e comunque non riferibili in modo esclusivo ad una precisa scuola di pensiero".

${ }^{13}$ Vd. F. Dümmler, nella recensione agli Epicurea di Usener, ristampata in F. DüMmLeR 1901: 316-318. Che l'opera sia rivolta contro gli Stoici è opinione di K. Ziegler 1965: 131-4; cf. D. Babut 1969: 62 ss.; G. Santese 1999: 44, n. 116.

${ }_{14}$ Ad esempio, la distinzione tra i vari tipi di è certamente epicurea (cf. Ep. ad Men. 127.8), ma anche platonica (Re. 558d-559c; Phdr. 254a-d). L'esempio della scrofa di Crommione (987F) riporta a Lach. 196d-e, dove è citato lo stesso aninale; 988B-C, dove si afferma che gli uomini affrontano pericoli solo per evitare rischi peggiori, può essere confrontato con Phd.68d-69e; 989C, dove è implicita l'idea che le città di mare sono particolarmente esposte alla corruzione dei costumi, può essere accostato a Pl. $\mathrm{Lg}$. 704d-707c; e in generale le osservazioni sugli effetti dell'irruzione delle esterne nell'anima, che evocano l'immagine della città invasa da un esercito straniero, riportano sicuramente alla nota tematica della Repubblica. Senza aggiungere, naturalmente, che tutta la tematica della discussione sulle virtù è spiccatamente di matrice socratico-platonica. 
filosofico che è eufemistico chiamare eclettico o sincretistico (in proposito magari mi soffermerò un'altra volta): ma non ha mai presentato veramente la voce di Ulisse. Di certo né l'autore né il lettore possono realmente condividere l'opinione di Grillo: questa può far sorridere in vari punti per la sua arditezza e per la sua stravaganza, ma alla fine si deve capire che l'autore ha lasciato al lettore il compito di proseguire nel cammino della riflessione in vista di una possibile conclusione. Come il suo maestro Platone, anche Plutarco si riserva di approfondire l'argomento e tirare le conclusioni nell'ambito dell'insegnamento orale svolto all'interno alla sua scuola. Come si può rispondere a Grillo, lo dovranno dire gli alunni - o forse anche i lettori stessi -, preparando una argomentazione seria, da opporre come adeguata antilogia.

In questa opera, certamente retorica (che molti ritengono giovanile ${ }^{15}$, perché fresca, divertente, inattesa, sorprendente, piena di bravura retorica), l'autore non vuole dimostrare una tesi seria, filosofica, coerente con tutto il suo pensiero complessivo di filosofo, ma solo proporre una provocazione retorica, un divertimento sofistico se vogliamo, una tesi paradossale, che dev'essere funzionale ad una vera riflessione filosofica sul tema della virtù animale.

${ }^{15}$ Cf. K. Ziegler 1965: 133; J. Bergua Cavero 1991: 17; W. C. Helmbold 1957: 490; G. Indelli 1995: 21; A. Barigazzi 1992: 301. Ma forse, in definitiva, ha ragione J. A. Fernández Delgado 2000: 181, che è totalmente scettico sulla cronologia di tutte le opere zoologiche di Plutarco. 


\section{Bibliografia}

Babвitт, F.C., Plutarch's Moralia, vol. IV, ed. with an English Translation, Cambridge-Mass., London 1972 (1935).

Babut, D., Plutarque et le Stoïcisme, Paris, 1969.

BARIgAzzI, A., "Implicanze morali nella polemica plutarchea sulla psicologia degli animali", in I. Gallo (ed.), Plutarco e le scienze. Atti del IV convegno plutarcheo, Genova-Bocca di Magra aprile 1991, Genova 1992: 297-315.

Becchi, F., "Biopsicologia e giustizia verso gli animali in Teofrasto e Plutarco", Prometheus 27, 2001: 119-135.

Becchi, F., "Irrazionalità e razionalità degli animali negli scritti di Plutarco, ovvero: il paradosso della superiorità razionale ed etica degli animali", Prometheus 26, 2000: 205-225.

Becchi, F., "Istinto e intelligenza negli scritti zoopsicologici di Plutarco", in M. Bandini; F.G. Pericoli (edd.), Scritti in memoria di D. Pieraccioni, Firenze, 1993: 59-83.

Bergua Cavero, J., "Cinismo, ironía y retórica en el Bruta ratione uti de Plutarco", in J. García López; E. Calderon Dorda (edd.), Estudios sobre Plutarco: paisaje y naturaleza. Actas del II Simposio Español sobre Plutarco, Murcia 1990, Madrid 1991: 13-19.

Cainzza, A., Plutarco, Conversazioni a tavola, libro II, Napoli, 2001.

Casanova, A., "Il 'Grillo' di Plutarco e la tradizione della figura di Ulisse", Ploutarchos n.s. 4, 2006/2007: 19-28.

Casanova, A., "Il 'Grillo' di Plutarco e Omero", in J. Boulogne (ed.), Les Grecs de l'antiquité et les animaux. Le cas remarquable de Plutarque, Lille, 2005: 97-109.

Casanova, A., "The Time Setting of the dialogue Bruta animalia ratione uti", in A. Pérez Jiménez; F. Titchener (edd.), Historical and Biographical Values of Plutarch's Works. Studies devoted to professor Philip A. Stadter by the International Plutarch Society, Málaga, Logan, Utah, 2005: 121-131.

Casanova, A., "Traduzioni e riusi del Grillo di Plutarco nel Rinascimento fiorentino: Machiavelli e Gelli", in A. Pérez Jiménez (ed.), Plutarco renovado. Importancia de las traductiones modernas de Vidas y Moralia, Málaga, 2010: 65-76.

Dierauer, U., Tier und Mensch im Denken der Antike. Studien zur Tierpsychologie, Anthropologie und Ethik, Amsterdam, 1977.

Dümmler, F., Kleine Schriften, I. Zur griechischen Philosophie, Leipzig,1901.

Fernández Delgado, J. A., "Le Gryllus, une éthopée parodique”, in L. van der Stockt (ed.), Rhetorical Theory and Praxis in Plutarch, Acta of the 
IV International Congress of the IPS, Leuven, july 3-6, 1996, Louvain, Namur, 2000: 171-181.

Helmbold, W. C.; Cherniss, H., Plutarch's Moralia, vol. XII, ed. with an English Translation, Cambridge, Mass., London 1957.

Hirzel, R., Der Dialog. Ein literarische Versuch, Leipzig, 1895.

Hubert, C., Plutarchi Moralia, vol. VI.1, Lipsiae, 1954.

Indelli, G., Plutarco, Le bestie sono animali razionali, Intr. testo critico trad. e comm., Napoli, 1995.

Inglese, L.; Santese, G., Plutarco, Il cibarsi di carne, intr. testo critico, trad. e commento, Napoli, 1999.

Jounan, F., Euripide et les légendes des Chants Cypriens, Paris, 1966.

Magini, D., Plutarco, Del mangiar carne. Trattati sugli animali, traduzione di Donatella M., con introduzione di D. Del Corno, Milano, 2001.

Russell, D., Plutarch. Selected Essays and Dialogues, A new translation by D. R., Oxford, New York, 1993.

Teodorsson, S.-T., A Commentary on Plutarch's Table Talks, vol. I (1-3), Göteborg, 1989.

Usener, H., Epicurea, Leipzig, 1887.

Ziegler, K., Plutarco, trad. it., Brescia 1965 (= "Plutarchos von Chaironeia", RE XXI.1, 1951: 636-962. 